(Aus dem physiologischen Institute zu Bonn.)

\title{
Kann der Dünndarm stearinsauren Kalk resorbieren?
}

Von

E. A. Knauer, Bonn.

Im Jahre 1901 hat Otto Loewi in einer Sitzung der Gesellschaft zur Beförderung der gesamten Naturwissenschaften zu Marburg $^{1}$ ) als Beweis für die Behauptung, dass zur Resorption der Fette ihre vorherige Verseichung nicht erforderlich sei, u. a. berichtet, er habe gefunden, dass in isolierten, sorgfältig gereinigten und beiderseits abgebundenen Darmschlingen Fettsäuren aus eingebrachten wasserunlöslichen Kalkseifen resorbiert würden. Dass weder diese von Loewi angestellten Versuche, noch auch seine übrigen in der genannten Abhandlung enthaltenen Ausführungen über die Theorie der Fettresorption als Gründe gegen die Lehre von der nur in gelöster Form sich vollziehenden Resorption der Fette irgendwie stichhaltig sind, ist im Jahre 1902 von Pflüger in einer Arbeit „Über Kalkseifen als Beweise gegen die in wässeriger Lösung sich vollziehende Resorption der Fette" ${ }^{2}$ ) ausführlich und schlagend dargelegt worden. Selbst wenn Loewi die Resorption der Kalkseifen bewiesen hätte, so bleibt er, wie Pflüger hervorhebt, den Beweis schuldig, dass diese Resorption in ungelöster Form verlaufen wäre; er behauptet, es sei kein Lösungsmittel für die Kalkseifen dagewesen, muss aber ihre Löslichkeit in Galle selbst zugeben. Allerdings experimentierte Loewi an einer abgebundenen, vorher gereinigten Darmschlinge. Er meint, dadurch die Mitwirkung der Galle ausgeschlossen zu haben. Dagegen führt Pflüger mit Recht an ${ }^{3}$ ), dass z. B. beim Hunde die innere Oberfläche des Dünndarms einem dichtesten Rasen gleicht, der von Galle und Schleim durchtränkt ist, dass ferner zahllose feine Röhrchen als Ausführungsgänge der

1) Sitzungsbericht Nr. 7, Juni 1901.

2) Pflüger's Archir Bd. 89 S. 211.

3) l. c. S. 218. 
Lieberkühn'schen Drüsen auch Gallenbestandteile enthalten können, und dass daher die von $\mathrm{L} 0 \mathrm{e}$ wi vorgenommene Reinigung der Darmschlingen die sichere Entfernung der Galle nicht verbürgt. Sieht man aber auch von der Galle als Lösungsmittel ab, so zeigt $\mathrm{P} f \mathrm{l}$ üg $\mathrm{er}^{1}$ ), dass sich Kalkseifen auch gegenüber $1 \%$ iger Sodalösung und gegenüber destilliertem Wasser nicht wie ganz unlösliche Körper verhalten. Man kann nämlich aus Kalkseifen, die man in verdünnter Sodalösung oder destilliertem Wasser aufgeschwemmt hat, mit Äther Fettsäuren ausschütteln. Daraus folgt, dass die Kalkseifen in Berührung mit Wasser hydrolytischer Spaltung fähig, also auch wahrscheinlich bis zu einem gewissen Grade löslich sind. Jedenfalls aber wird das im Darmsaft der abgebundenen Darmschlinge enthaltene Natriumkarbonat mit den durch Hydrolyse aus den Kalkseifen frei gewordenen Fettsäuren lösliche Seifenmoleküle erzeugen können. Loewi's Grundvoraussetzung, dass die Kalkseifen im Safte der abgebundenen Darmschlinge absolut unlöslich sind, ist also nicht zutreffend.

Würde demgemäss eine Resorption der Kalkseifen, wenn sie wirklich stattfände, nicht das beweisen, was durch sie bewiesen werden soll, so ist auf der anderen Seite auch die Thatsache einer Resorption nicht als sicher feststehend anzusehen. Die von Pflüger gegen die Versuche Loewi's erhobenen Bedenken sind so wahr und haben sich durch meine nachfolgend mitgeteilten Untersuchungen so fast Wort für Wort bestätigt, dass ich am besten die betreffenden Sätze aus Pflüger's Erwiderung hier nochmals zum Abdruck bringe. Pflüg er sagt S. 217 a. a. O.:

"Loewi hat bei der Beschreibung seiner Versuche nicht angegeben, an welchem Tiere sie angestellt wurden, welcher Teil des Darmes zur Aufnahme der Kalkseifen gewählt worden ist, $o b$ die ganze Masse oder nur ein Teil der Kalkseifen resorbiert wurde, wie er die Resorption nachwies und auf welchem Wege die angewandten Kalkseifen dargestellt worden sind, so dass sicher alle löslichen Seifen ausgeschlossen waren."

Und weiter S. 217 und 218 a. a. O.:

„Zuerst wäre die Frage zu behandeln, ob die Gesamtmasse der injizierten Kalkseifen oder nur ein Teil derselben resorbiert worden ist. Wenn man erwägt, dass in dem Kot unter den für die Resorption günstigsten Bedingungen, d. h. unter normalen Verhältnissen,

1) 1. c. S. 222 . 
also trotz Mitwirkung der Galle Kalk- und Magnesiaseifen enthalten sind, kann man mit Sicherheit annehmen, dass bei dem Versuch von Loewi die ganze Menge der in die Darmschlinge injizierten Kalkseife nicht resorbiert worden ist." „Ohne jeden Zweifel kann aber diese ganze Menge auch dann nicht wiedergefunden werden, wenn nicht die Spur von Kalkseife resorbiert worden ist. Die Kalkseifen bilden unendlich feine Pulver, die in Wasser aufgeschwemmt, durch fast alle Filter gehen. Dieser unendlich feine Staub wird z. B. beim Hunde nach der Injektion in den durch die Zottenfäden des Dünndarms gebildeten dichten Rasen eindringen und durch späteres Ausspülen, ja Auspinseln, nicht vollständig wiedergewonnen werden können. Es wäre also als Kontrolle dieser Versuche nötig gewesen, die Injektion der Kalkseifen in eine tote Darmschlinge $\mathrm{zu}$ machen, diese länger $\mathrm{zu}$ kneten und dann $\mathrm{zu}$ versuchen, wie viel man von der injizierten Kalkseife durch Ausspülen wiedergewinnen kann. Loewi giebt nicht an, ob er solche Kontrollversuche gemacht hat. - Die Anwendung chemischer, zugleich die Darmwand beeinflussender Mittel zur Wiedergewinnung der Kalkseife hat auch ihre bedenkliche Seite."

Die Frage der Resorbierbarkeit oder Nichtresorbierbarkeit der Kalkseifen durfte also durch die Versuche Loewi's nicht als erledigt angesehen werden, und Herr Geheimrat Pflüger betraute mich daher mit der Aufgabe, den Gegenstand unter Berücksichtigung aller im Vorstehenden angedeuteten Vorsichtsmassregeln einer Nachprüfung zu unterziehen. Diese Untersuchung habe ich bereits im Jahre 1902 ausgeführt und abgeschlossen, und zwar nur dank der einzig dastehenden, weder Mühe noch Zeit scheuenden Hilfe des Herrn Professor M. Bleibtre u-Greifswald, des damaligen I. Assistenten des physiologischen Institutes zu Bonn. Leider wurde die vorliegende Veröffentlichung durch eine einjährige Seereise, die ich inzwischen unternahm, bis heute verzögert.

\section{Anfertigung des Präparates.}

Bei meinen Versuchen benutzte ich die Kalkseife der Stearinsäure, die folgendermassen dargestellt wurde:

Aus chemisch reiner Stearinsäure wurde zunächst nach dem von Pflüger in seiner Abhandlung: „Über die Bedeutung der Seifen

1) Dieses Archiv Bd. 88 S. 442. 
für die Resorption der Fette" ${ }^{1}$ ) angegebenen Verfahren eine reine Natronseife dargestellt. Dasselbe besteht im Wesentlichen darin, dass man zuerst die Stearinsäure in heissem Alkohol löst und dann mit alkoholischer Natronlauge in geringem Überschuss auf dem Wasserbade verseift. Der Alkohol wird nun verjagt, der Rückstand in heissem Wasser gelöst und aus der heissen Lösung durch Einfliessenlassen in chemisch reine gesättigte Kochsalzlösung die Seife ausgesalzen. Die abfiltrierte Seife wird durch gesättigte Kochsalzlösung gewaschen und mit einer kräftigen Filterpresse ausgepresst. Den entstandenen Seifenkuchen pulverisiert man und löst ihn in heissem Alkohol. Filtriert man jetzt die heisse Lösung, so bleibt das Kochsalz auf dem Filter zurück. Beim Erkalten scheidet sich die Seife aus dem Filtrat aus. Man presst sie mit der Filterpresse wieder stark aus und erhält schliesslich die Natronseife in Gestalt harter Blättchen.

Um die Natronseife in die Kalkseife überzuführen, lösten wir sie in heissem Wasser und liessen die heisse Lösung unter beständigem Umrühren in eine reichliche Menge destillierten Wassers einfliessen, das einen Überschuss von chemisch reinem Chlorcalcium enthielt. Die Kalkseife wurde abfiltriert, der Niederschlag vom Filter genommen, noch einmal mit reinem destilliertem Wasser verrieben, wieder filtriert, aufs Neue ausgepresst und zur sicheren Beseitigung des Chlorids der ausgepresste Kuchen noch einmal zerkleinert, in destilliertem Wasser verrieben und ausgepresst. Das ablaufende Wasser gab jetzt keine Chlorreaktion mehr.

Der harte Seifenkuchen ist leicht zu pulverisieren. Das Pulver scheint dem Augenschein nach vollkommen trocken, enthält aber noch etwas anhaftendes Wasser.

Es wurde zuerst ein Präparat $A$ angefertigt, welches bei den ersten Versuchen verwandt wurde, als es aufgebraucht war, ein Präparat B. In dem Präparat A wurde das anhaftende Wasser zunächst belassen, weil eine absolute Trockenheit für den Zweck der Versuche nicht nötig war; Präparat B wurde dagegen im Vacuum vollständig getrocknet. Vor dem Gebrauch wurden nun beide Präparate auf ihre Reinheit geprüft.

\section{Prüfung des Präparates auf seine Reinheit.}

Wir bestimmten in den Präparaten den Gehalt an Fettsäure und an Kalk. 


$$
\text { Präparat A. }
$$

a) $1,0545 \mathrm{~g}$ des Pulvers werden im Scheidetrichter mit Wasser aufgeschwemmt und nach Ansäuern mit Salzsäure fünfmal bis zur Erschöpfung mit Äther ausgeschüttelt.

Wir erhalten $0,9485 \mathrm{~g}$ Stearinsäure.

b) Der wässerige Rückstand wird mit $\mathrm{NH}_{3}$ ammoniakalisch gemacht und nach den Vorschriften von Fresenius ${ }^{1}$ ) mit Ammoniumoxalat als oxalsaurer Kalk gefällt. Die Fällung glühen wir im Platintiegel vor dem Gebläse bis zur Gewichtskonstanz. Das entstandene $\mathrm{CaO}$ wiegen wir und finden: $0,0932 \mathrm{~g} \mathrm{CaO}$.

Im Calciumstearat verbinden sich $0,9485 \mathrm{~g}$ Stearinsäure mit $0,0935 \mathrm{~g}$ Calciumoxyd. Der gefundene Wert erreicht den verlangten also mit der wünschenswertesten Genauigkeit. Kalk.

$0,9485 \mathrm{~g}$ Stearinsäure entspricht nun $1,012 \mathrm{~g}$ stearinsaurem

Die $1,0545 \mathrm{~g}$ des Pulvers enthalten mithin: $1,012 \mathrm{~g}$ reines Calciumstearat.

Der Rest: $0,0425 \mathrm{~g}$ ist Wasse $\mathrm{r}$, da andere Beimengungen bei der Art der Darstellung nicht wohl vorhanden sein können.

In dem Präparat A sind also neben reinem stearinsaurem Calcium noch $4,03 \%$ Wasser.

$$
\text { Präparat } B \text {. }
$$

a) Nachdem das Präparat auf konstantes Gewicht gebracht war, wurden $1,036 \mathrm{~g}$ des Pulvers wieder im Scheidetrichter aufgeschwemmt, mit Salzsäure angesäuert und mit Äther bis zur Erschöpfung ausgeschüttelt.

Es fand sich: $\mathbf{0 , 9 6 9 1} \mathrm{g}$ Stearinsäure. 1,036 $\mathrm{g}$ Calciumstearat sollten enthalten: $\mathbf{0 , 9 7 1} \mathrm{g}$. Die Differenz beträgt also nur $0,0019 \mathrm{~g}$ oder $0,2 \%$ der zu erwartenden Stearinsäuremenge.

b) Zur Bestimmung des Kalkes sollte wieder wie bei Präparat A die ausgeschüttelte wässerige Lösung dienen. Da aber die Analyse verunglückte, wurde eine neue Probe des Pulvers genommen, im Platintiegel verascht und vor dem Gebläse bis zur Gewichtskonstanz geglüht, wie bei einer Kalkbestimmung.

$1,0033 \mathrm{~g}$ des Pulvers hinterliessen dabei $0,0929 \mathrm{~g}$ Asche, die

1) Fresenius, Quantitative Analyse, 6. Aufl. S. 235 f. Braunschweig 1898. 
nur aus $\mathrm{CaO}$ bestehen kann. Die zu verlangende Menge $\mathrm{CaO}$ beträgt $0,0927 \mathrm{~g}$; also fast vollständige Übereinstimmung.

Der Sicherheit halber wurde die Asche aber noch einmal in Salzsäure aufgelöst, in ein Becherglas übergespült, wieder mit $\mathrm{NH}_{3}$ und Ammoniumoxalat behandelt und der Kalk als Calciumoxyd bestimmt. Wir erhielten: $\mathbf{0 , 0 9 3 8} \mathrm{CaO}$.

Wir können das Präparat also als vollkommen reinen, wasserfreien, stearinsauren Kalk ansehen.

\section{Die Versuchsmethodik.}

Es sieht so aus, als sei nichts leichter, wie eine bestimmte Menge der Substanz, deren Resorbierbarkeit oder Nichtresorbierbarkeit festgestellt werden soll, in eine vorher gereinigte Darmschlinge einzubringen und nach geeigneter Zeit nachzusehen, wie viel übrig geblieben ist. Bei der praktischen Ausführung stellen sich aber alle die Schwierigkeiten ein, die, wie ich schon in der Einleitung hervorhob, P flüger vorausgesagt hat und die durch die eigentümliche Beschaffenheit der gepulverten Kalkseifen bedingt werden. In trockenem Zustande haben diese Pulver die unangenehme Eigenschaft, beim geringsten Anlass sehr stark zu stäuben. Mit Wasser aufgeschwemmit, kriechen sie an den feuchten Wänden empor und setzen sich allenthalben in der hartnäckigsten Weise fest. Bei dem Versuch, die Substanz quantitativ genau in den Darm hinein- und wieder herauszubringen, macht sich das in der unliebsamsten Weise bemerkbar. Was zunächst die exakte Überführung einer genau abgewogenen Menge Kalkseife in das Darmstück betrifft, so könnte man ja daran denken, die nach Behandlung mit der Filterpresse erhaltenen klingharten Seifenstücke zu wiegen und in das Darmrohr einzulegen. Das würde sehr leicht ohne Verlust gelingen. Davon haben wir aber von vornherein abgesehen; denn wenn dabei eine Resorption nicht beobachtet würde, so könnte man das mit Recht auf die zur Resorption ungeeigneten Seifenstücke schieben. Um die Seife in hinreichend ausgedehnte Berührung mit den resorbierenden Flächen des Darmes zu bringen, ist ihre Pulverisierung unerlässlich. Damit wird aber auch die Gefahr, durch das starke Stäuben des Pulvers erhebliche Verluste zu erleiden, sehr gross. Die Substanz mit Wasser in den Darm hineinzuspülen, führt zu dem Übelstand, dass das Pulver an den Wänden des Gefässes, aus dem man es in 
den Darm bringen will, trotz allen Nachspülens hartnäckig hängen bleibt. Noch mancher andere Weg wurde ausprobiert und wieder aufgegeben, ehe wir zu dem bei den späteren.Versuchen angewandten Verfahren gelangten, das jeden Verlust beim Einbringen der Substanz in das Darmstück ausschliesst.

Ein $v$-förmig gebogenes Glasrohr von solchen Dimensionen, dass es bequem in den Darm einzuführen war und die einzuführende Menge Substanz gut aufnehmen konnte, wurde zuerst leer, dann mit Substanz beschickt, gewogen. Nachdem die gewählte Dünndarmschlinge ${ }^{1}$ ) an beiden Enden durchschnitten und mit sehr reichlichen Mengen Wasser ausgespült war, wurde das eine Ende der Darmschlinge durch eine Ligatur verschlossen, in das andere der eine Schenkel des $v$-förmigen Glasrohres eingeschoben und mit einer Ligatur fest eingebunden. Den anderen Schenkel verbanden wir mit einem Gummischlauch. Durch Druck auf einen Gummiballon, der an dem freien Ende des Schlauches angebracht wurde, oder auch durch einfaches Blasen mit dem Munde gelang es leicht, fast das ganze Pulver in die Darmschlinge zu befördern. Noch während der Ballon zusammengepresst war bezw. mit dem Munde der Blasedruck auf seiner Höhe gehalten wurde, wurde der Schlauch nahe dem Glasröhrenende mit einer Klemmpinzette gefasst und zur Sicherheit auch unterbunden. Dann wurde der übrige Teil des Schlauches abgeschnitten, die Darmschlinge reponiert und die Bauchhöhle wieder geschlossen. Das Glasröhrchen fixierten wir in der Naht.

Bei diesem Verfahren bleibt eine verhältnismässig nur sehr geringe Substanzmenge an den Innenwänden des Röhrchens haften. Diese rechnen wir nachher zu der im Darm verbliebenen, nicht resorbierten Substanz hinzu und sind so sicher, dass auch kein Stäubchen beim Einbringen des Pulvers verloren ging. Dass nicht die ganze abgewogene Substanz in den Darm gelangt, ist helanglos, da sich herausstellte, dass wir obnehin viel mehr hineingebracht hatten, als die etwa vorhandene Resorptionsfähigkeit des Darmes bewältigen könnte. Zudem stellte der im Glasröhrchen verbliebene Rest ersichtlich nur einen ganz kleinen Bruchteil des Ganzen dar.

Das hier geschilderte Verfahren des Einbringens der Substanz erschien uns im Verlaufe der Versuche als das zweckmässigste; in

1) Wo in den Versuchen etwas Besonderes nicht bemerkt ist, wählten wir ein Stück des mittleren Dünndarmdrittels. 
den ersten Versuchen wurde anders vorgegangen, worüber das Nähere bei der Beschreibung der Versuche selbst berichtet wird.

Damit war für den ersten Teil unserer Aufgabe, die Substanz ohne Verlust in den Darm zu bringen, endlich eine befriedigende Lösung gefunden. Noch weit grössere Schwierigkeiten harrten unser bei dem zweiten Teil, bei der Frage, wie bekommen wir nach dem Versuche die Substanz wieder quantitativ exakt aus dem Darme heraus? (Wir nehmen schon hier vorweg, dass nach dem Versuch überhaupt noch Substanz in der Darmschlinge vorhanden war, und zwar dem Augenschein nach die ganze Substanz.) Man könnte sich nun vorstellen, es gelänge durch kräftiges Durchspülen des Darmrohres mit Wasser, die übriggebliebene Substanz wieder zu gewinnen. Wenn man so verfährt, erhält man in der That einen grossen Teil der eingebrachten Kalkseife zurück. Wenn man sich aber damit begnügen wollte, so wäre freilich nichts einfacher, als einen Verlust an stearinsaurem Kalk zu konstatieren, aber nichts verkehrter, als diesen Verlust einer Resorption zuzuschreiben. Schneidet man nämlich jetzt das Darmstück der Länge nach auf, so sieht man, dass die ganze Schleimhaut noch mit anhaftenden Seifenteilchen bedeckt ist, und noch so energisches Ausspulien mit Wasser wird diese Reste nicht vollständig von der Darmwand entfernen können. Daher bleibt nichts anderes übrig, als zu mechanischen Hilfsmitteln seine Zuflucht zu nehmen; wir bedienten uns dazu eines weichen Pinsels. Mit Hilfe desselben und wieder mit sehr viel Spülwasser gelingt es denn, die dem Darm anhaftenden Seifenteile in eine grosse Schale zu sammeln. Das erfordert aber eine drei- bis vierstündige, ausserordentlich mübsame Arbeit; immer aufs neue entrleckt man Seifenteilchen in den Falten der Schleimhaut, und die Eigensehaft des Seifenpulvers, an allen feuchten Flächen entlang zu kriechen, führt dazu, dass nach dem Aufschneiden des Darmes nicht bloss an der Innenfläche, sondern auch an der Serosafläche die weissen Stäubchen erscheinen. Ja, schliesslich müssen nicht nur die benutzten Instrumente, sondern auch die Hände des Experimentators aufs sorgfältigste von Substanzpartikelchen befreit werden, die unversehens und trotz aller Vorsicht auf die Haut gelangen. Dabei kann man nicht umhin, zum Spülen viele Liter Wasser zu verbrauchen, so dass nichts anderes übrigbleibt, als diese grossen Wassermassen in einer weiten Porzellanschale, die schliesslich die ganze Substanz möglichst ohne Verlust enthält, auf einem Wasserbade zu kleinem Volumen einzudampfen. Dann muss die Substanz 
wieder mit gleicher Sorgfalt in einen grossen Scheidetrichter hinübergespült werden, wobei wir darauf achten, dass nicht mehr wie ca. 1/2 bis ${ }^{2 / 3}$ Liter Flüssigkeit in den Scheidetrichter gelangen. Dann wird nach Ansäuren mit Salzsäure die Flüssigkeit bis zur Erschöpfung mit Äther extrahiert, der Ätherextrakt gesammelt und im Trockenschrank bei 50 bis $60^{\circ}$. und hierauf im Exsiccator bis zur Gewichtskonstanz getrocknet.

Verwandten wir somit auf das quantitative Sammeln der der Darmwand anhaftenden Seifenteilchen die grösste Mühe und vermieden so wohl grössere Verluste, so möchten wir doch bestimmt behaupten, dass kleine Verluste an Substanz immer noch vorgekommen sind. Es wäre daher nicht zu verwundern gewesen, wenn der gewonnene Ätherextrakt etwas hinter der in der Kalkseife in den Darm gebrachten Stearinsäure zurückgeblieben wäre. Indessen stellte sich jetzt heraus, dass das sorgfältige Herauspinseln der Substanz aus der Schleimhaut einen anderen Übelstand im Gefolge hat; es ist nämlich gar nicht zu vermeiden, dass nicht bloss Darmsekret, sondern auch Darmepithelieu und andere Gewebsteile mit herausgespült und herausgepinselt werden. Diese Beimengungen enthalten aber Fett, Fettsäuren, Seifen und andere in Äther lösliche Körper. Diese geben beim Ausschütteln mit Äther in saurer Reaktion natürlich ebenfalls Extrakt ab, der sich der aus unserer Kalkseife stammenden Fettsäure beimischt. Um dem zu begegnen, könnte man daran denken, schon vor dem Ansäuren mit Salzsäure mit Äther auszuschütteln, und so die als Verunreinigung beigemengten Neutralfette zu entfernen. Nachdem aber P fl ü g e r nachgewiesen hat, dass die in Wasser aufgeschwemmten Kalkseifen sich ständig in hydrolytiseher Dissoziation befinden und beim Ausschütteln mit Äther erhebliche Mengen Fettsäure abgeben, muss man auch a priori annehmen, dass dieses Verfahren nicht bloss das als Verunreinigung anhaftende Fett beseitigt, sondern auch einen Teil der gesuchten Stearinsäure. In der Tat haben wir beim dritten Versuch, als wir einmal probeweise vor dem Ansäuren mit Äther ausschüttelten, feststellen können, dass der erhaltene Ätherextrakt zum grössten Teile aus Stearinsäure bestand. Im übrigen haben wir aber infolge der aus Darmsekret und Darmgewebe stammenden Beimengung von Ätherextrakt in allen unseren Versuchen nachher mehr Ätherextrakt im Darminhalt gefunden, als der mit der Kalkseife eingeführten Fettsäure entsprach. Die kleinen Verluste, die beim Sammeln der Kalkseifen 
wohl immer noch vorgekommen sind, werden also durch diesen Gewinn an Ätherextrakt überkompensiert.

Wenn endlich eine Resorption der eingeführten Seife, einschliesslich des Kalkes, stattfände, so müsste diese Resorption auch durch die Abnahme des Kalkes in der Darmschlinge nachzuweisen sein. Aber da die Sekrete und Gewebe des Darmes ebenfalls Kalksalze enthalten, so liegen die Dinge hier genau wie bei der Stearinsäure. Ja, unsere Versuche zeigen, dass die Zunahme an Calcium verhältnismässig viel grösser ist als die an Ätherextrakt.

Angesichts dieser Verhältnisse war es erst recht geboten, mit den eigentlichen Resorptionsversuchen Kontrollversuche in der von Pflüger vorgeschlagenen Art zu verbinden. Das geschah bei dem dritten und vierten der unten mitgeteilten Versuche. Diese Kontrollversuche wurden ganz in der Art wie die eigentlichen Versuche an einer angrenzenden Darmschlinge angestellt, nachdem der Hauptversuch heendigt und das Tier getötet worden war. Auch in diesen Kontrollversuchen wurde ein Mehr an Ätherextrakt und ein Mehr an Kalk gefunden, gerade wie im Hauptversuch.

Dass nun der Ätherextrakt grösstenteils aus Stearinsäure bestand, ging schon aus seinem Aussehen hervor, derselbe war kristallisiert wie Stearinsäure, nur durch die Verunreinigung mehr oder weniger verfärbt. Es war daher vorauszusehen, dass der Schmelzpunkt nur annähernd dem reiner Stearinsäure entsprechen würde. Was die Bestimmung der Schmelzpunkte betrifft, so ist zu bemerken, dass der Ätherextrakt, der durch Abdampfen des Äthers in Glasschalen gewonnen wurde, sich nicht homogen abschied; beim $A b-$ dunsten des $\ddot{A}$ thers schieden sich zuerst die mehr verunreinigten und mehr verfärbten Partien $a b$ und bildeten eine Randschicht in der Schale, dann folgten in der Tiefe der Schale mehr weisse Zonen.

Entsprechend war denn auch der Schmelzpunkt nicht an allen Stellen gleich. Meist wurden daher von jedem Ätherextrakt drei Schmelzpunkte genommen, von der Randzone, der mittleren Zone und der Bodenpartie. Erstere hatte in der Regel den niedrigsten, letztere den höchsten Schmelzpunkt. Die beobachteten Schmelzpunkte gingen herab bis auf $65^{\circ}$, gegenüber dem der Stearinsäure von $69,2^{\circ}$. 


\section{Die Versuche.}

1. Versuch. Kaninchen. 26. Februar 1902.

Die Substanz wurde in einem mit Kappenschliff versehenen Wägeröhrchen abgewogen und dieses Röhrehen nach Abnahme der Kappe unter Führung eines etwas weiteren, zuerst eingelegten Metallrohres in den Darm eingeführt. Das Metallröhrchen endigte in drei Zinken, die die Darmwände etwas auseinanderhalten und so ein erweitertes Lumen schaffen sollten. Das Glasröhrchen wurde mit dem offenen Ende bis zu diesem Zinken vorgeschoben, und es sollte nun das Pulver in den etwas erweiterten Raum der Darmhöhle hineingeschüttet werden. Unter allmählichem Zurückziehen beider Röhrchen wollten wir so nach und nach den ganzen Inhalt in das Darmstück einfüllen. Indessen funktionierte dieses Verfahren nicht ganz in der gewünschten Weise, indem der Darm sich ventilartig in die Öffnung des Metallrohres hineinzustülpen strebte und so das Herausfallen der Substanz hinderte; auch berührte die Darmwand trotz Führung durch die Metallröhre das Ende des Glasrohres und benetzte dieses mit Darmsekret. Dadurch drohte beim Zurückwägen einerseits ein schwer zu eliminierender Fehler, anderseits war es für das Seifenpulver eine gefährliche Gelegenheit, sich auf der Aussenwand des Glasröhrchens festzukleben. Die zuerst gewählte Darmschlinge konnte daher nicht zum Versuch benutzt werden, sondern diente nur zur Einübung des Verfahreus. Es gelang aber schliesslich an einer anderen Stelle des Dünndarmes, die Substanz mit nur unerheblichen Verlusten einzuführen. Die eingebrachte Menge wurde durch Zurückwägen des Wägeröhrchens gefunden. Das vorher mit Wasser ausgespülte und beiderseits abgebundene Darmstück war ca. $25 \mathrm{~cm}$ lang. Die Schleimhäute zeigten normale alkalische Reaktion.

Das Gewicht der eingebrachten Substanz vom Präparat A betrug $0,8315 \mathrm{~g}$. Die langen, fruchtlosen Bemühungen an der erst gewählten Darmschlinge hatten die ganze Operation in die Länge gezogen, und diesem Umstande ist es wohl zuzuschreiben, dass das Tier 3/4 Stunden nach Einbringen des Pulvers verendete. Infolge dieser kurzen Wirkungsdauer verliert der Versuch an Wert. Da sein Ergebnis aber in anderer Beziehung lehrreich ist, teilen wir ihn doch mit. 
Nach dem Versuch wurde die Darmschlinge herausgeschnitten und der Darm der Länge nach gespalten. Das Seifenpulver war durch das ganze Darmstück verteilt und lag da - für das Auge unverändert.

Der Darminhalt, nach dem im vorigen Abschnitt III geschilderten Verfahren untersucht, enthielt

$$
0,762 \mathrm{~g} \text { Ätherextrakt. }
$$

In den eingeführten $0,8315 \mathrm{~g}$ Substanz sind nach der oben mitgeteilten Analyse $\quad 0,748 \mathrm{~g}$ Stearinsäure.

Nach dem Versuch ist also in dem Darmstück ein Plus von $0,014 \mathrm{~g}$ Ätherextrakt

zu verzeichnen. Das sind $1,9 \%$ der in den Darm eingebrachten Stearinsäure.

Der Ätherextrakt sieht genau aus wie krystallisierte Stearinsäure, nur ist er der Verunreinigung entsprechend von gelblicher Farbe. Seine Schmelzpunkte an den verschiedenen Stellen der Schale waren $66,8^{0} ; 66,8^{\circ} ; 67,0^{\circ}$.

2. Versueb. Kaninchen. 28. Februar 1902.

Die Substanz wurde bei diesem Versuch in die Darmschlinge eingeblasen. Ein $v$-förmiges, beiderseits offenes Glasröhrchen diente als Wägeglas. Das eine Ende war mit einem Gummischlauch versehen, das andere wurde in die Darmöffnung eingeführt. Durch den Schlauch wollten wir die Substanz in den Darm einblasen, dann sollte das Glasrohr herausgezogen, an den Aussenwänden abgewischt und zurückgewogen werden, während das Darmende durch eine Ligatur verschlossen wurde. Dieses Verfahren hatte aber den Übelstand, dass schon sofort beim Aufhören des Blasens, sowie nachher bein Herausziehen des Glasrohres ein Rückwärtsstäuben des Pulvers stattfand. Bei der zuerst gewählten Darmschlinge schienen die durch Stäuben entstandenen Verluste so beträchtlich, dass wir dieses Darmstück verliessen und ein anderes.wählten, bei dem das Einbringen der Substanz ohne nennenswerten Verlust gelang.

Die in den Darm gebrachte Quantität war $0,6155 \mathrm{~g}$ vom Präparat A. Das entspricht $0,5536 g$ Stearinsäure und $0,0544 \mathrm{~g}$ Calciumoxyd.

Nach $3^{1 / 2}$ Stunden wurde das Tier getötet, das benutzte $a b-$ gebundene Darmstück herausgeschnitten, und nun verfubren wir wieder in der im vorigen Abschnitt geselilderten Weise. 
Im Darminhalt fand sich $0,5727 \mathrm{~g}$ Ätherextrakt statt eingebrachter . . 0,5536 "Stearinsäure. Mithin ein Plus von. $0,0191 \mathrm{~g}$ Ätherextrakt oder $3,4 \%$ der eingeführten Stearinsämre.

Der Ätherextralit ist wieder krystallisiert wie Stearinsäure, aber gelblich bis bräunlich gefärbt wegen anhaftender Verunreinigungen.

Als Schmelzpunkte an den verschiedenen Stellen der den Ätherextrakt enthaltenden Schale wurden gefunden die Zahlen $66,5^{\circ}$ (am Rand, wo die Braunfärbung am stärksten war); $66,7^{\circ} ; 66,9^{\circ}$.

In dem Darmiuhalt wurde auch der Kalk als $\mathrm{CaO}$ bestimmt. (Der als $\mathrm{CaO}$ gewogene Glührückstand war etwas gelblich gefärbt; vermutlich sind geringe Mengen Eisen mitgewogen worden.)

Wir fanden . . 0,0582 $\mathrm{g}$ statt . . . . 0,0544 "Calciumoxyd

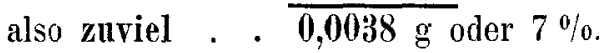

Nach der Wägung des Glührückstandes wurde derselbe übrigens mit einer abgemessenen Menge $1 / 10 \mathrm{n}$. Salzsäure aufgelöst und der Überschuss an Säure zurücktitriert; darnach ergab sich $0,0596 \mathrm{~g}$ $\mathrm{CaO}$, in befriedigender Übereinstimmung mit der durch Gewicht ermittelten Menge.

Die Darmschleimhaut reagierte zu Beginn des Versuches normal alkalisch.

3. Versuch. Kaninchen. 23. Mai 1902.

Da das Wägeglas mit Präparat A öfter geöffnet, Substanz daraus entnommen und wieder zurückgefüllt worden war, schien es uns nicht mehr absolut sicher, dass der Wassergehalt unserer Kalkseite noch derselbe war; wir zogen es daher ror, das Pulver nummehr ganz trocken zu machen. Es wurde bei $50-60^{\circ}$ im Trockenstand schliesslich auf konstantes Gewicht gebracht.

Bei dieser Untersuchung wurde ausser dem Versuch am lebenden Darm auch ein Kontrollversuch am toten Darm vorgenommen, $d . h$. nachdem der eigentliche Resorptionsversuch beendet war, das Tier getötet und die benutzte Darmschlinge herausgeschnitten worden war, wurde ganz genau so mit einer anderen Düundarmschlinge verfahren, Substanz in dieselbe eingeführt, die in situ belassene Schlinge eine Zeit lang geknetet und auch diese Darmschlinge nach Verlauf einiger Stunden herausgeschnitten und in derselben Weise wie bei der ersteren auf ihren Inhalt untersucht. 
Dann wurde der ganze Dünndarm herausgenommen und gemessen. Folgendes Schema gibt ein Bild davon, um welche Darmabschnitte es sich handelte.

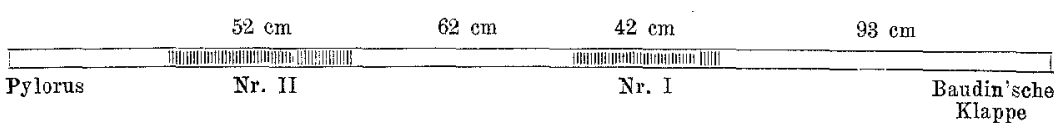

Es war die Absicht gewesen, das Kontrollstück Nr. II näher an das zum eigentlichen Versuch dienende Stück Nr. I heranzulegen, es wurde aber bei seiner verwickelten Lage in der Bauchhöhle die Entfernung unrichtig geschätzt.

Nr. I. Der eigentliche Resorptionsversuch.

Die Einbringung der Substanz geschah nach dem im Abschnitt III beschriebenen Verfahren. Eingeführt wurde in die $42 \mathrm{~cm}$ lange Darmschlinge 0,7597 $\mathrm{g}$ Kalkseife, entsprechend 0,712 g Stearinsäure und 0,0702 g CaO. Da es etwas schwierig war, den Darm ganz zu reponieren und um zu viele Manipulationen zu vermeiden, nähten wir die Bauchwunde nur teilweise zu und bedeckten den Bauch des aufgebundenen Tieres mit feuchtwarmen Kompressen. In dieser Lage hielten wir das Tier $3^{1 / 2}$ Stunden lang. Sein Zustand war augenseheinlich ständig gut. Kurz vor Abschluss des Versuches überzengten wir uns, dass im zugehörigen Stück des Mesenteriums gute Zirkulation vorhanden war: eine angeschnittene Mesenterialarterie spritzte kräftig. Das Darmstück war etwas aufgetrieben vom Einblasen der Substanz, war aber, wie sich nachher erwies, grösstenteils mit Flüssigkeit angefüllt. Die Schleimhaut reagierte alkalisch.

Nachdem wir nun in der üblichen mühsamen Weise den Inhalt des Darmes möglichst quantitativ genau in den Scheidetrichter gebracht, versuchten wir zuerst, wieviel Ätherextrakt sich ohne vorheriges Ansäuren mit Salzsäure bloss durch Ausschütteln mit Äther gewinnen liesse. $\mathrm{Zu}$ bemerken ist, dass die im Scheidetrichter befindliche Flüssigkeit auf Lackmus eben merklich sauer reagierte.

Durch mehrmaliges Ausschütteln bloss mit Äther (ohne zu erschöpfen) erhielten wir $0,1615 \mathrm{~g}$ Ätherextrakt. Derselbe war krystallisiert und bestand offenbar, wenn auch verunreinigt, grösstenteils aus Stearinsäure. Der Schmelzpunkt war $65^{\circ}$.

Dann folgte nach Ansäuern mit Salzsäure die Ausschüttelung mit Äther bis zur Erschöpfung, und diese ergab noch $0,5949 \mathrm{~g}$ Ätherextrakt. 
Als Schmelzpunkte dieser zweiten Portion fanden wir die Zahlen:

an den Randpartien: $65,7^{\circ}$ bezw. $66,1^{\circ}$,

in der Tiefe der Sclale: $67,0^{\circ}$ bezw. $67,0^{\circ}$.

Im Ganzen erhielten wir also . . . . $\mathbf{0 , 7 5 6 4} \mathbf{g}$ Ätherextrakt.

Die mit der Substanz eingeführte Stearin-

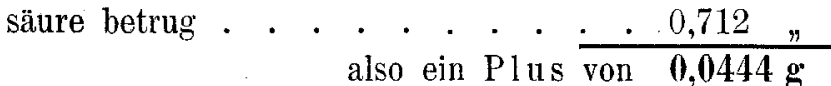

d. i. $6,2 \%$ der eingeführten Stearinsäure.

Die Bestimmung des Kalkes ergab . . . 0,1041 g: CaO

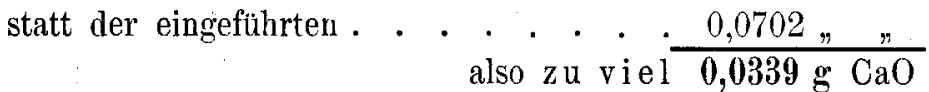

oder $48 \%$ der eingeführten Kalkmenge. Auch hier war der Glührückstand etwas gelblich gefärbt.

Nr. II. Der Versuch an der toten Darmsehlinge.

In das $52 \mathrm{~cm}$ lange Darmstück wurden eingebracht $0,750 \mathrm{~g}$ stearinsaurer Kalk, entsprechend $0,703 \mathrm{~g}$ Stearinsäure und $0,0694 \mathrm{~g} \mathrm{CaO}$.

Die Seife blieb $2^{3 / 4}$ Stunden in dem Darm, der inzwischen mehrfach bewegt und geknetet wurde.

Es wurde im Darminhalt gefunden . . . $0,7679 \mathrm{~g}$ Ätherextrakt, statt der mit der Substanz eingeführten . . 0,7030 "Stearinsäure, also ein Plus von $0,0649 \mathrm{~g}$ Ätherextrakt, d. i. $9,2 \%$ der eingeführten Stearinsäuremenge.

Der Schmelzpunkt wurde an den verschiedenen Stellen übereinstimmend $\mathrm{zu} 65,5^{\circ}$ bestimmt.

Bei der Kalkbestimmung erhielten wir . . . . 0,0978 g $\mathrm{CaO}$ Mit der Kalkseife waren eingeführt worden . . . 0,0693 " "

d. i. $\mathrm{zu}$ viel $\overline{0,0285 \mathrm{~g}} \mathrm{CaO}$ oder $41 \%$ der eingeführten Kalkmenge.

4. Versuch. Hund (mittelgrosser schwarzer Pudel).

In Chloroform-Äthernarkose wird eine Dünndarmschlinge freigelegt, an beiden Enden durchschnitten und mit viel Wasser ausgespült. Das $v$-förmige Rohr hat für den Hund etwas grössere Verhältnisse. Es wird wieder neben dem eigentlichen Resorptionsversuche Nr. I ein Kontrollversuch Nr. II an einer benachbarten Darmschlinge gemacht. Über die Lage der gewählten Darmstücke belehrt folgendes Schema: 


\begin{tabular}{|c|c|c|c|}
\hline $44 \mathrm{em}$ & $36 \mathrm{~cm}$ & $36 \mathrm{~cm}$ & $100 \mathrm{~cm}$ \\
\hline Pylorns & Nr. I & Nr. II & $\begin{array}{c}\text { Baudin'sche } \\
\text { Klappe }\end{array}$ \\
\hline
\end{tabular}

Die Stücke I und II liegen also unmittelbar nebeneinander.

Nr. I. Der eigentliche Resorptionsversuch.

In das Stück Nr. I werden $1,2400 \mathrm{~g}$ stearinsauren Kalks vom Präparat B, entsprechend $1,1621 \mathrm{~g}$ Stearinsäure eingeblasen. Man könnte einwenden, durch das Aufblasen des Darmes und die Spannung der Darmwand würden abnorme, für die Resorption ungünstige Verhältnisse geschaffen. Bei diesem Versuch verfuhren wir daher noch so, dass nach dem Einblasen des Pulvers der Gummischlauch zwar sofort durch eine Klemmpinzette in der Mitte verschlossen wurde, dass dann aber das freie Ende in ein zum Teil mit Wasser gefülttes Kölbchen bis unter den Wasserspiegel getaucht und die Klemmpinzette für wenige Augenblicke geöffnet wurde. Dabei strömt die eingepresste Luft teils schon von selbst durch die Elastizität der Darmwand, teils auf sachtes Zusammendrücken derselben wieder heraus, nimmt dabei allerdings eine Staubwolke von Substanz mit, die aber nicht verloren geht, sondern auf dem Wasser des Kölbchens schwimmt und nachher zu dem vielen Waschwasser, mit dem nach dem Versuch der Darm ausgespült wird, hinzukommt; ebenso wurden die an den Wänden des abgeschnittenen Schlauchstückes haftenden Seifenteilehen gesammelt.

Nachdem die Bauchwunde sorgfältig zugenäht war, wurde der Hund losgebunden; zunächst schlief er noch eine halbe Stunde, erholte sich dann wieder und war bald wieder ganz munter. $3^{1 / 2}$ Stunden nach Einbringen der Substanz töteten wir das Tier und schnitten das benutzte Darmstück heraus. Dasselbe wurde noch sehr sorgfältig vom anhaftenden Mesenterium und besonders von allen sichtbaren Fettbestandteilen frei präpariert. Das eine Ende wurde dann geöffnet und vom anderen Ende aus, in dem noch das Glasrohr mit einem kurzen Stück Schlauch steckte, Wasser durchgespült und zwar in diesem Falle literweise. Nichtsdestoweniger fand sich nachher beim Aufschneiden des Darmes noch sehr viel anhaftende Substanz, die wieder mühsam mit Finsel und Wasser gesammelt wurde.

Wir gewannen aus dem Darminhalt . . 1,3322 g Ätherextrakt Mit der Substanz waren eingeführt worden 1,1621 "Stearinsäure also ein Plus von . . . . . . . . $\overline{0,1701} \mathrm{~g}$ Ätherextrakt oder $14,7 \%$ der eingeführten Stearinsäuremenge. 
Die Schmelzpunkte des Ätherextrakts waren: in den Randpartien $66^{\circ}$, in der Mitte $66,3^{\circ}$, am Boden $67^{\circ}$. Die Schleimhaut zeigte zu Beginn des Versuches normale, alkalische Reaktion.

Nr. II. Kontrollversuch an der toten Darmschlinge.

Gleich nach Entfernung des Darmstückes No. I wurde auch der ganze übrige Darm aus dem Kadaver herauspräpariert, die Längenverhältnisse gemessen und ein $35 \mathrm{~cm}$ langes Stück unmittelbar unter No. I für den Kontrollversuch ausgewählt. Das Einbringen der Substanz geschah genau sỏ wie bei No. I.

Es wurde in No. II eingeführt: 1,2281 g Calciumstearat entsprechend 1,151 g Stearinsäure.

Und nun wurde die Darmschlinge mehrmals geknetet und durch aufgelegte feuchtwarme Tücher bei Körpertemperatur gehalten. Die Substanz verblieb im Darm $21 / 2$ Stunden.

Es fand sich im Darminhalt . 1,243 g Ätherextrakt gegenüber eingebrachten • • · 1,151 n Stearinsäure also ein Plus von . . . . . $0,092 \mathrm{~g}$ oder $8 \%$ der eingeführten Stearinsäure.

Die Schmelzpunkte des Ätherextraktes waren: Randpartie $65,2^{\circ}$; Mitte $65,1^{\circ} ;$ Bodenpartie $66,2^{\circ}$.

\section{Ergebnisse.}

Die aus den Versuchen gewonnenen Zahlen finden sich in folgender Tabelle (S. 106) zusammengestellt.

Wir sehen, तass in allen Fällen nach dem Versuch eine grössere Menge Ätherextrakt vorgefunden wird, wie der mit der Kalkseife eingebrachten Stearinsäuremenge entspricht. Das Mehr wechselt, von kleinen Werten bis zu recht beträchtlichen.

Schon der Augenschein lehrte, dass der wiedergefundene Ätherextrakt zum grössten Teil aus Stearinsäure bestand, das zeigen auch die Schmelzpunkte, die zwar in allen Fällen unter dem der reinen Stearinsäure lagen, sich demselben aber sehr näherten.

In allen Fällen lag, auch schon für das blosse Auge erkennbar, der grösste Teil der eingeführten Kalkseifen offenbar noch unverändert im Darm da. Dabei handelt es sich in keinem der Ver- 
E. A. Knauer:

\begin{tabular}{|c|c|c|c|c|c|c|}
\hline \multirow{2}{*}{ 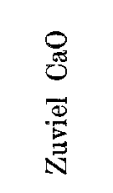 } & $\Xi$ & 1 & L & $\stackrel{\infty}{\leftrightarrow}$ & $F$ & 1 \\
\hline & $\begin{array}{l}\infty \\
. \nexists\end{array}$ & 1 & $\begin{array}{l}\infty \\
8 \\
8 \\
0 \\
0\end{array}$ & 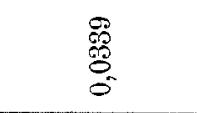 & $\begin{array}{l}28 \\
00 \\
0 \\
0 \\
0\end{array}$ & 1 \\
\hline \multicolumn{2}{|c|}{ 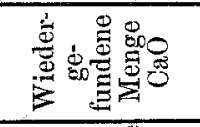 } & 1 & $\begin{array}{l}0 \\
\infty \\
: \\
8 \\
0\end{array}$ & 행 & 兽 & 1 \\
\hline \multicolumn{2}{|c|}{ 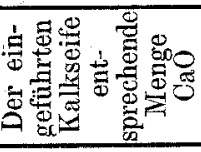 } & - & 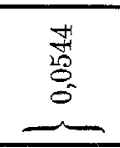 & $\begin{array}{r}\begin{array}{c}0 \\
0 \\
0 \\
0 \\
0\end{array} \\
\end{array}$ & $\begin{array}{rr}g_{0}^{8} \\
8 \\
0 \\
0\end{array}$ & $\underbrace{1}$ \\
\hline \multicolumn{2}{|c|}{ 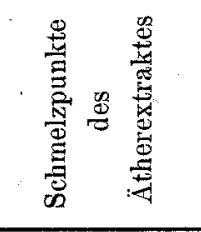 } & 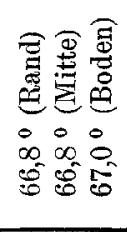 & 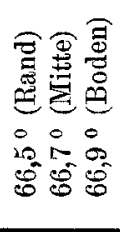 & 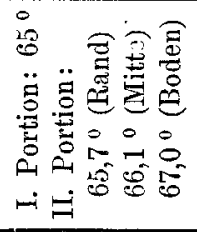 & 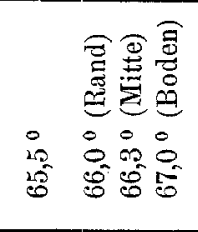 & 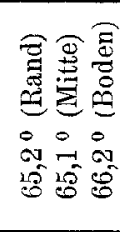 \\
\hline \multirow{2}{*}{ 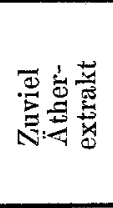 } & $\begin{array}{l}\circ \\
\end{array}$ & $\vec{a}$ & 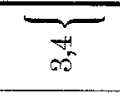 & जे & $0 \quad \underset{ \pm}{ \pm}$ & $\underbrace{}_{\infty}$ \\
\hline & 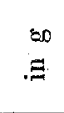 & $\begin{array}{l}\vec{H} \\
0 \\
0\end{array}$ & $\begin{array}{l}\vec{\sigma} \\
\dot{\sigma} \\
\dot{\sigma}\end{array}$ & $\begin{array}{l}\text { 苦 } \\
8 \\
8\end{array}$ & $\begin{array}{l}\stackrel{9}{0} \\
\dot{8} \\
8\end{array}$ & : \\
\hline \multicolumn{2}{|c|}{ مo } & 赵 & 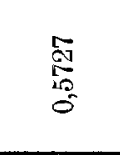 & 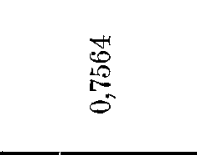 & 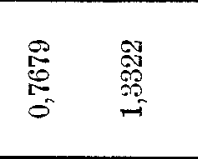 & $\stackrel{\text { I }}{\mathrm{N}}$ \\
\hline \multicolumn{2}{|c|}{ 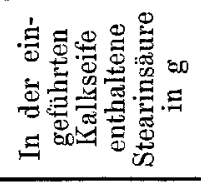 } & $\frac{\substack{0 \\
\mathbb{N} \\
0}}{0}$ & 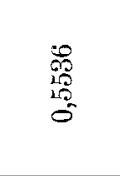 & 愛 & 量 & $\stackrel{5=1}{=10}$ \\
\hline \multicolumn{2}{|c|}{ 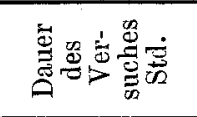 } & $\overrightarrow{00}$ & $\stackrel{\overrightarrow{0}}{\vec{\partial}}$ & $\stackrel{-\infty}{\infty}$ & $\stackrel{0}{\infty}_{\infty}^{+1}$ & $\stackrel{s}{-\pi}$ \\
\hline \multicolumn{2}{|c|}{ 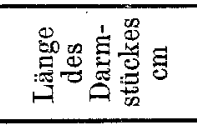 } & 19 & a. & $\overbrace{}^{\text {g }}$ & 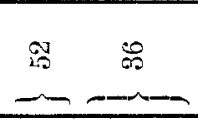 & $\stackrel{\infty}{\infty}$ \\
\hline \multirow{2}{*}{\multicolumn{2}{|c|}{ 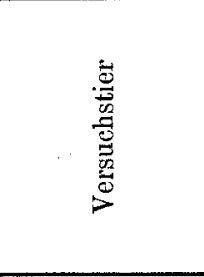 }} & \multirow{2}{*}{ 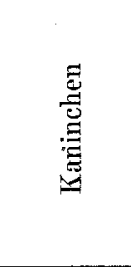 } & \multirow{2}{*}{ 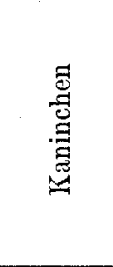 } & 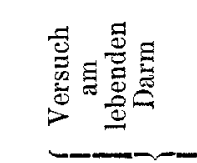 & 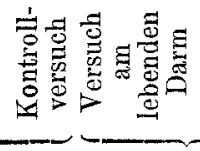 & 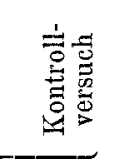 \\
\hline & & & & 窝 & $=$ & \\
\hline$\dot{\vec{z}}$ & & 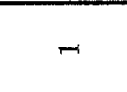 & or & $\infty$ & & \\
\hline
\end{tabular}


suche um, absolut genommen, grosse Fettsäuremengen, so dass die dem Darm gestellte Resorptionsaufgabe über seine Kräfte ginge; besonders bei dem Hunde wäre eine Resorption von wenig mehr als $1 \mathrm{~g}$ Fettsäure bei dem grossen Resorptionsvermögen des Hundedarms für Fett für ein Darmstück von ca. ${ }^{1 / 3} \mathrm{~m}$ Länge gewiss keine hohe Leistung gewesen. Wenn überhaupt Kalkseifen resorbiert werden, hätte also sehr wohl die ganze eingeführte Menge verschwunden sein können. Statt dessen finden wir das Seifenpulver ganz unverändert im Darme liegend vor, natürlich mit Darmsekret vermischt. Niemals war auch nur die geringste Injektion der Chylusgefässe, wie man sie sonst nach Resorption von Fetten sieht, zu beobachten. Bei der chemischen Untersuchung des Darminhalts nach dem Versuch führten die im III. Abschnitt erörterten Verbältnisse zu einer Vermehrung des Ätherextraktes durch die in Äther löslichen Bestandteile, die mit dem Darmsekret sowie aus den Geweben der Darmwand bei dem unvollkommenen mechanischen Zusammensuchen der allenthalben anhaftenden Kalkpartikelchen hineingeraten waren. In noch höherem Masse zeigte sich in den Fällen, wo wir daraufhin untersuchten, der Kalk vermehrt.

Bei den in zwei Fällen vorgenommenen Kontrollversuchen beobachteten wir dieselbe Vermehrung, zwar, wie leicht zu verstehen, nicht genau um dieselben Prozentzahlen, aber um Grössen von derselben Bedeutung. Im Versuch Nr. 3 ist die Vermehrung beim Kontrollversuch grösser ausgefallen, in Nr. 4 beim Hauptversuch.

Das Endergebnis unserer Untersuchung können wir folgendermassen fassen:

Trotz aller Bemühungen ist es uns nicht gelungen, eine Resorption von Kalkseifen aus lebenden, abgebundenen Darmschlingen nachzuweisen. Wenn eine solche stattfindet, so kann sie nur sehr geringfügig: sein.

Leider erlaubten die technischen Schwierigkeiten nicht, durch unsere Untersuchung die Frage scharf zur Entscheidung zu bringen, ob vielleicht in ganz geringem Umfang eine Resorption des stearinsauren Kalkes stattfindet oder nicht. Jedenfalls sind wir aber berechtigt, nach unseren Versuchen die Behauptung, der Darm vermöge stearinsauren Kalkzu resorbieren, so lange in $Z$ weifel zu ziehen, als dieselbe nicht mit besseren Beweisen gestützt wird. 
108 E. A. Knauer: Kann der Dünndarm stearinsauren Kalk resorbieren?

Ich habe die Ehre, Herrn Geh. Rat E. Pflüger für die gütige Anregung zu der vorliegenden Arbeit meinen ehrerbietigsten Dank auszusprechen. Ganz besonders eng und herzlich verpflichtet füble ich mich aber Herrn Professor M. Bleibtreu-Greifswald gegenüber, ohne dessen ungewöhnlich weitgehende Unterstützung mir die Durehführung dieser Untersuchung nie gelungen wäre. 